Localizador: 19016 doi: $10.35366 / 93981$

\title{
Esclerodermia sistémica juvenil en una lactante: reporte de caso
}

\author{
Juvenile systemic sclerosis in an infant: case report
}

\author{
Alexandra Romero Flores, ${ }^{*}$ Noemí Chávez Dávila, ${ }^{\ddagger}$ Marcelo Toro Arias, ${ }^{\S}$ María Cecilia Vivar ${ }^{\|}$
}

\footnotetext{
Palabras clave:

Esclerosis, sistémica,

juvenil.

Keywords:

Sclerosis, systemic, juvenile.
}

\section{* Hospital Pedítrico «Baca Ortiz», Especialista en Dermatología. \\ ₹ Universidad Central del Ecuador, Médico Postgradista de segundo año de Dermatología. \\ ${ }^{\S}$ Hospital Pediátrico «Baca Ortiz», Especialista en Anatomía Patológica. " Universidad Central del Ecuador, Médico Postgradista de tercer año de Anatomía Patológica. \\ Conflicto de intereses: Ninguno.}

Recibido: 04/Abril/2019. Aceptado: 30/Julio/2019

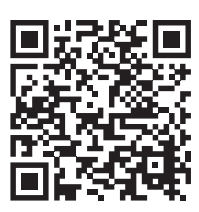

\section{REsumen}

La esclerodermia sistémica juvenil es una rara patología crónica del tejido conectivo, cuyos síntomas comienzan, en promedio, a los 11 años de edad. Tiene una alta morbilidad y mortalidad, y es una enfermedad sobre la que no existen consensos de tratamiento, por lo que se convierte en un reto para los especialistas que la manejan. Este trabajo describe un caso de esclerodermia juvenil sistémica difusa en una niña de un año, diagnosticada y tratada de forma multidisciplinaria en el Hospital Pediátrico «Baca Ortiz» de Quito, Ecuador. Destaca la importancia de mantener una alta sospecha, para llegar a un diagnóstico precoz, y realizar tratamientos farmacológicos y no farmacológicos para estabilizar la actividad de la enfermedad y mejorar la calidad de vida de las personas afectadas por esta enfermedad.
ABSTRACT

Juvenile systemic scleroderma is a rare chronic pathology of connective tissue, whose symptoms begin on average at age 11 . It has a high morbidity and mortality, being a disease of which there is no consensus of treatment, so it becomes a challenge for the specialists who manage it. This paper describes a case of diffuse systemic juvenile cutaneous scleroderma in a one-yearold girl, diagnosed and treated in a multidisciplinary manner at the «Baca Ortiz» Children's Hospital in Quito, Ecuador, which highlights the importance of maintaining a high level of suspicion, allowing early diagnosis, and carrying out pharmacological and non-pharmacological treatments to stabilize the activity of the disease and improve the quality of life of people affected by this disease.

\section{INTRODUCCIÓN}

$\amalg$ a esclerodermia es una enfermedad crónica \lrcorner del tejido conectivo caracterizada por la afectación autoinmune, vasculopatía y fibrosis. ${ }^{1}$ No hay datos epidemiológicos reales sobre su incidencia mundial; sin embargo, un estudio realizado en el Reino Unido e Irlanda muestra una baja incidencia, con un medio de inicio de la enfermedad a los 11 años. ${ }^{2}$

La forma predominante de la esclerosis infantil es la esclerodermia localizada, mientras que la sistémica es poco frecuente, pero su mayor morbimortalidad es el manejo de la información.

A continuación, presentamos el caso de una paciente de un año un mes de edad, quien fue diagnosticada con esclerodermia sistémica juvenil difusa (ESJcd), en el Hospital Pediátrico «Baca Ortiz». Recibió el tratamiento multidisciplinario, y logró la estabilización de la enfermedad y la mejoría de su calidad de vida.

\section{PRESENTACIÓN DEL CASO}

Preescolar de un año un mes, con antecedente de infección urinaria y gastrointestinal a los seis y siete meses de edad, respectivamente.

Acude con su madre quien refiere que desde los 10 meses de edad ha sufrido pérdida de peso, dificultad para la prensión de objetos, sedestación, deambulación y hace dos meses ha tenido dificultad para la deglución de sólidos, resequedad en la piel y lesiones pruriginosas en las piernas que dejan manchas blanquecinas.

Al examen físico se evidencia una lactante mayor en mal estado general, FC: $150 \mathrm{lat} / \mathrm{min}$, T: $36.4{ }^{\circ} \mathrm{C}$, antropometría: peso $7.2 \mathrm{~kg}$ (P-3er), longitud $71 \mathrm{~cm}(\mathrm{P}-3 \mathrm{er}), \mathrm{IMC} 14.28$ (P-2do), PC: $44 \mathrm{~cm}$ (P: 2do) que corresponden a retraso pondoestatural y psicomotor.

Pelo, cejas y pestañas finas y escasas, dificultad para la apertura bucal, piel atrófica, brillante, turgente, acartonada con pérdida de pliegues fisiológicos, máculas y manchas hipopigmentadas 
de bordes irregulares bien definidos de predominio en tórax y extremidades; además, presencia de placas eritematosas e induradas que comprometen manos y pies bilateral, con disminución de la movilidad, y pápulas eritematosas que forman placas en extremidades inferiores predominantes en la cara externa de los muslos (Figura 1).

Con la sospecha clínica de Esclerodermia Sistémica Juvenil (ESJc) ya que cumplía el mayor de los criterios preliminares del Consenso de Paediatric Rheumatology European Society (PRES), the American College of Rheumatology (ACR), y la European League Against Rheumatism (EULAR) (esclerosis proximal a las articulaciones metacarpofalángicas o metatarsofalángicas), además de dos menores (esclerodactilia y disfagia), por lo que se solicitaron exámenes complementarios y valoración interdisciplinaria.

En los exámenes de laboratorio anticuerpos antinucleares (ANA) y anti Scl-70 fueron positivos. En imagen, el esofagograma reportó reflujo gastroesofágico grado III, ausencia de esclerosis y compresiones extrínsecas

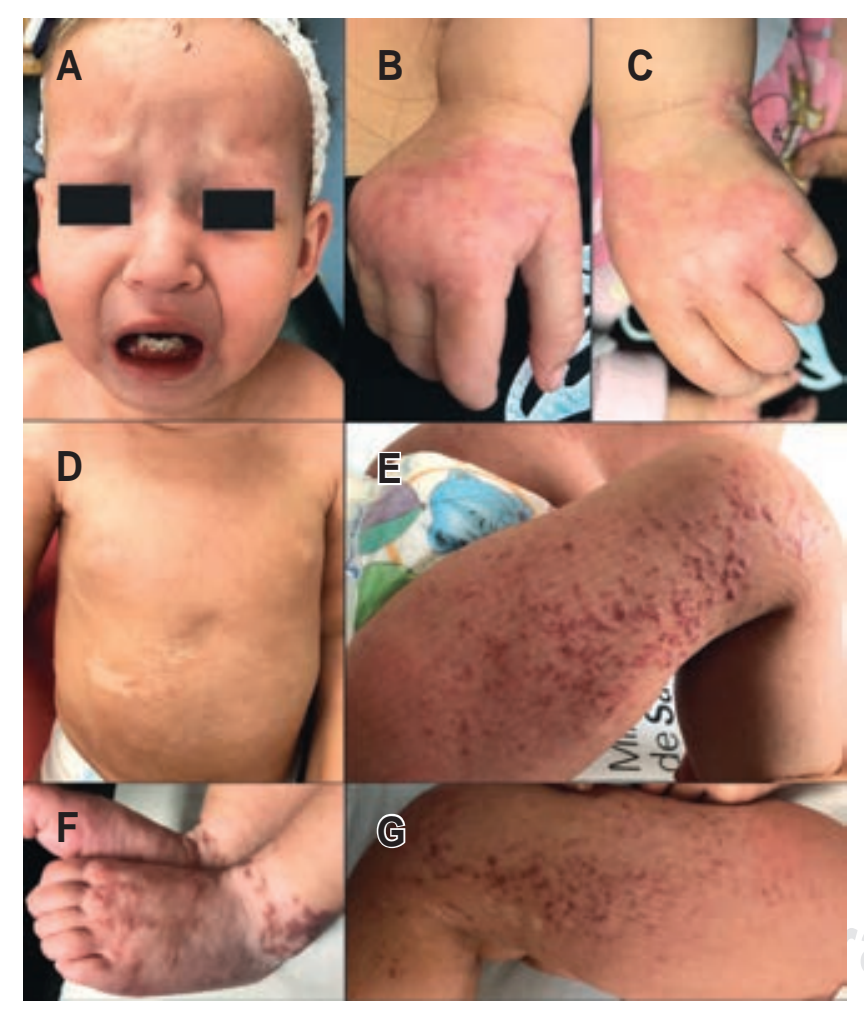

Figura 1: Imagen clínica inicial. A) Facies pálida, cejas y pestañas finas y escasas. B, C y F) Placas eritematosas, induradas. D) Máculas y manchas hipopigmentadas de bordes irregulares bien definidos, con áreas de atrofia, piel brillante, turgente, acartonada en tórax. $\mathbf{E}$ y $\mathbf{G}$ ) Pápulas eritematosas que forman placas en cara externa de muslos.
(Tabla 1). El estudio histopatológico de una biopsia cutánea de las lesiones indicó atrofia de glándulas ecrinas, con incremento de haces de colágeno que reemplazan el tejido celular subcutáneo positivo para tricrómico de Masson (Figura 2). Se corroboró y clasificó a la patología como esclerodermia sistémica juvenil difusa (ESJcd), por cumplir el criterio mayor y tres menores (a los mencionados previamente se suman: reflujo gastroesofágico y pruebas serológicas positivas).

Al confirmar el diagnóstico se inició tratamiento con corticoide tópico y sistémico, prednisona a $2.5 \mathrm{mg} / \mathrm{kg} / \mathrm{d}$ ía se mantuvo la dosis un mes, posteriormente se añadió metotrexato a $0.5 \mathrm{mg} / \mathrm{kg} / \mathrm{semanal}$ y ácido fólico iniciando retiro progresivo de prednisona hasta $5 \mathrm{mg} /$ día $(0.5 \mathrm{mg} /$ $\mathrm{kg} /$ día), llegando a la estabilización de la enfermedad, con controles periódicos en los que no se ha evidenciado alteración de las pruebas de función renal o hepática, ni compromiso de otro órgano interno. Además, recibió suplementos vitamínicos por su estado nutricional.

Luego de ocho meses de inicio del tratamiento, la paciente se alimenta sin dificultad incrementando su peso a $8.9 \mathrm{~kg}$ y el IMC a 14.4 (P 16) dentro de la normalidad, ha disminuido la esclerosis de un score de Rodman modificado de 12 a 6, mejorando la movilidad y fuerza muscular, camina sin apoyo, aunque tiene un retraso en el desarrollo psicomotor manejado con rehabilitación. Estos cambios muestran una mejoría en su calidad de vida (Figura 3).

\section{DISCUSIÓN}

La esclerodermia es una enfermedad autoinmune del tejido conectivo caracterizada por autoanticuerpos, vasculopatía y fibrosis. ${ }^{1}$ La forma juvenil tiene una incidencia estimada de 0.27 casos en un millón de niños anualmente, según un estudio en Reino Unido e Irlanda realizado de 2005 a 2007.2

La causa de la esclerodermia sistémica no se conoce claramente, sin embargo, algunos estudios muestran que esta patología podría presentarse en individuos con una predisposición genética o expuestos a factores ambientales específicos como virus, toxinas y/o medicamentos. ${ }^{3}$ Esto Ileva a una activación inmune, con el subsecuente daño endotelial y una producción excesiva de componentes de la matriz extracelular en la que los fibroblastos desempeñan un papel clave. ${ }^{4,5}$

Según los criterios preliminares del Consenso de la PRES, ACR y EULAR, un paciente menor de 16 años puede clasificarse dentro de la Esclerodermia Sistémica Juvenil (ESJc) si cumple con el criterio mayor (indispensable) y al menos dos de los 20 criterios menores (Tabla 2). 
Tabla 1: Laboratorio e imagen.

\begin{tabular}{|c|c|c|c|c|c|}
\hline & Mayo 2018 & Julio 2018 & Agosto 2018 & Noviembre 2018 & Febrero 2019 \\
\hline Biometría hemática & $\mathrm{N}$ & $\mathrm{N}$ & & & $\mathrm{N}$ \\
\hline Función renal & $\mathrm{N}$ & $\mathrm{N}$ & & & $\mathrm{N}$ \\
\hline Glucosa & $\mathrm{N}$ & $\mathrm{N}$ & & & $\mathrm{N}$ \\
\hline AST & $293(\uparrow 5 \mathrm{~N})$ & $33.4 \mathrm{~N}$ & & & $\mathrm{~N}$ \\
\hline ALT & $450(\uparrow 11 \mathrm{~N})$ & $22.7 \mathrm{~N}$ & & & $\mathrm{~N}$ \\
\hline LDH & $\mathrm{N}$ & & & & \\
\hline PCR & & $\mathrm{N}$ & & & $\mathrm{N}$ \\
\hline CPK & $\mathrm{N}$ & & & & \\
\hline Función tiroidea & $\mathrm{N}$ & & & & \\
\hline FR & & $\mathrm{N}$ & & & \\
\hline C3 & $\mathrm{N}$ & & & & \\
\hline $\mathrm{C} 4$ & $\mathrm{~N}$ & & & & \\
\hline ANA & $1 / 80$ & & & & \\
\hline Anti Scl-70 & Pos $24(<20)$ & & & & \\
\hline Anti-Ro & Neg & & & & \\
\hline Anticentrómero & Neg & & & & \\
\hline Anti-DNA & Neg & & & & \\
\hline Paratohormona & $\mathrm{N}$ & & & & \\
\hline Esofagograma & & & $\begin{array}{l}\text { Sin esclerosis n com- } \\
\text { presiones extrínsecas, } \\
\text { *RGE GIII }\end{array}$ & & \\
\hline RX tórax & & & Sin alteración & & \\
\hline ECO abdomen superior & & & Sin alteración & & \\
\hline Ecocardiograma & & & & $\begin{array}{l}\text { CIA ostium secundum } 1.3 \\
\text { x } 2.5 \mathrm{~mm} \text {, insuficiencia } \\
\text { tricúspidea leve, presión } \\
\text { sistólica en arteria pulmonar } \\
26 \text { mmHg, función sistólica } \\
\text { biventricular conservada }\end{array}$ & \\
\hline
\end{tabular}

AST = aspartato aminotransferasa, ALT = alanina aminotransferasa, LDH = lactato deshidrogenasa, PCR = reacción en cadena de polimerasa, CPK = creatina fosfocinasa, $\mathrm{ANA}=$ anticuerpos antinucleares, $\mathrm{CIA}=$ comunicación interauricular, $\mathrm{N}=$ normal, $\mathrm{Neg}=$ negativo, Pos $=$ positivo, $\mathrm{RGE}=$ reflujo gastroesofágico.

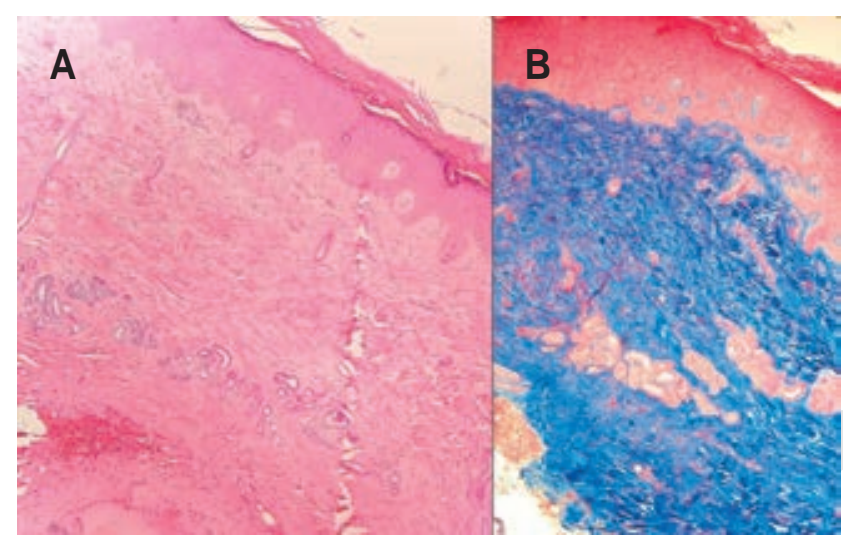

Figura 2: Biopsia cutánea. A) Hematoxilina eosina x4. Atrofia de glándulas ecrinas y anexos cutáneos. B) Incremento de haces de colágeno y fibrosis en la dermis superficial y profunda, que se pintan con azul en la coloración de tricrómico de Masson.

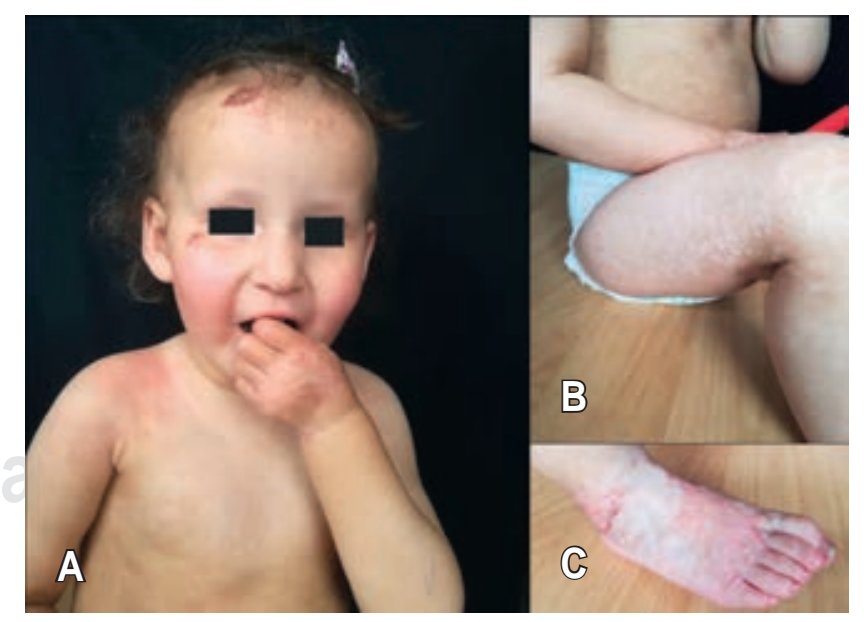

Figura 3: Imagen clínica de control. A) Mejor apertura bucal. B) Manchas hipopigmentadas de bordes irregulares bien definidos, piel adelgazada atrófica en tórax y extremidades. C) Esclerosis en pie. 
Hay tres subtipos principales de ESJc: difusa, limitada y superpuesta.

Esclerodermia sistémica juvenil difusa (ESJcd): caracterizada por afectación cutánea distal a los codos, rodillas y cuello, afectación cutánea de extremidades proximales, tórax y/o abdomen, progresión rápida y afectación de un órgano visceral principal (tracto gastrointestinal, pulmón más que el corazón y el riñón).

Esclerodermia sistémica juvenil limitada (ESJcl): presenta afectación cutánea distal a codos, rodillas y cuello, vasculopatía prominente (incluye fenómeno de Raynaud, hipertensión arterial pulmonar [HAP] y crisis renal), además de anticuerpos asociados como Antitopoisomerasa (ATA) y anticuerpo anticentrómero (ACA).

Esclerodermia sistémica juvenil superpuesta: tiene características de otras enfermedades reumáticas como dermatomiositis/polimiositis, lupus eritematoso sistémico, síndrome de Sjögren y artritis inflamatoria. Puede tener un patrón cutáneo limitado o difuso y se ve más frecuentemente en ESJc que en adultos. ${ }^{5}$

En el caso presentado la paciente cumplió con el criterio mayor del consenso de PRES, ACR y EULAR (esclerosis proximal a las articulaciones metacarpofalángicas o metatarsofalángicas), y tres criterios menores (esclerodactilia, reflujo gastroesofágico y pruebas serológicas positivas ANA y SCL-70); para ESJc la afectación cutánea tiene un patrón distal a los codos, rodillas, extremidades proximales, tórax y abdomen, progresión inicial rápida y compromiso gastrointestinal, por lo que se diagnosticó de ESJcd.

Para el manejo de los pacientes es fundamental determinar la gravedad de la patología, para lo que se dispone del puntaje de severidad de esclerodermia sistémica juvenil (J4S) (Tabla 3), que tiene un aspecto multidisciplinar en el que se incluyen nueve categorías de sistemas de órganos, puntuadas de 0 a 4, ponderando la participación de varios sistemas de órganos en función de su importancia clínica. ${ }^{6}$ La niña tuvo una puntuación inicial de 7 , la misma que disminuyó a 4.5 luego de ocho meses de tratamiento; sin embargo, esta escala presenta algunos parámetros que la paciente no cumple según los hitos propios de la edad, por lo que su fiabilidad disminuye.

El tratamiento es multidisciplinario, debe abarcar un manejo no farmacológico y farmacológico. Las medidas no farmacológicas incluyen el cuidado cutáneo con aplicación de emolientes, ejercicios para mantener la capacidad funcional, protección vascular y evitar desencadenantes como frío y trauma, junto al uso de férulas correctivas en caso de contracturas. Además, la esfera psicosocial debe ser manejada, junto con la educación del paciente y la familia, para ayudar a comprender la complejidad de la enfermedad, guiar la toma de decisiones y facilitar la adherencia terapéutica y no terapéutica. ${ }^{7}$

En el manejo farmacológico no existen ensayos controlados en niños, por lo que se basa en los datos de adultos, éste debe ser individualizado. ${ }^{7}$ Para ello se toman en consideración dos enfoques: el primero dirigido a modificar la enfermedad, con el fin de controlar el proceso subyacente de la patología en el que se incluyen corticoides, inmunomoduladores y antifibróticos, e incluso biológicos. En el caso presentado se trató a la paciente con prednisona y metotrexato, con mejoría y estabilización de la enfermedad hasta el momento. El segundo enfoque se debe dirigir a los órganos diana afectados, en este caso el gastrointestinal, que presenta reflujo gastroesofágico y debe ser manejado en forma similar a los pacientes sin esclerodermia, con modificación del estilo de vida y la dieta. Los inhibidores de la bomba de protones (IBP) son los fármacos más eficaces para el tratamiento de reflujo gastroesofágico en esclerodermia debiendo utilizarlos en pacientes sintomáticos, ya que las terapias a largo plazo

\section{Tabla 2: Criterios preliminares de esclerodermia sistémica juvenil (ESJc) PRES, ACR y EULAR.}

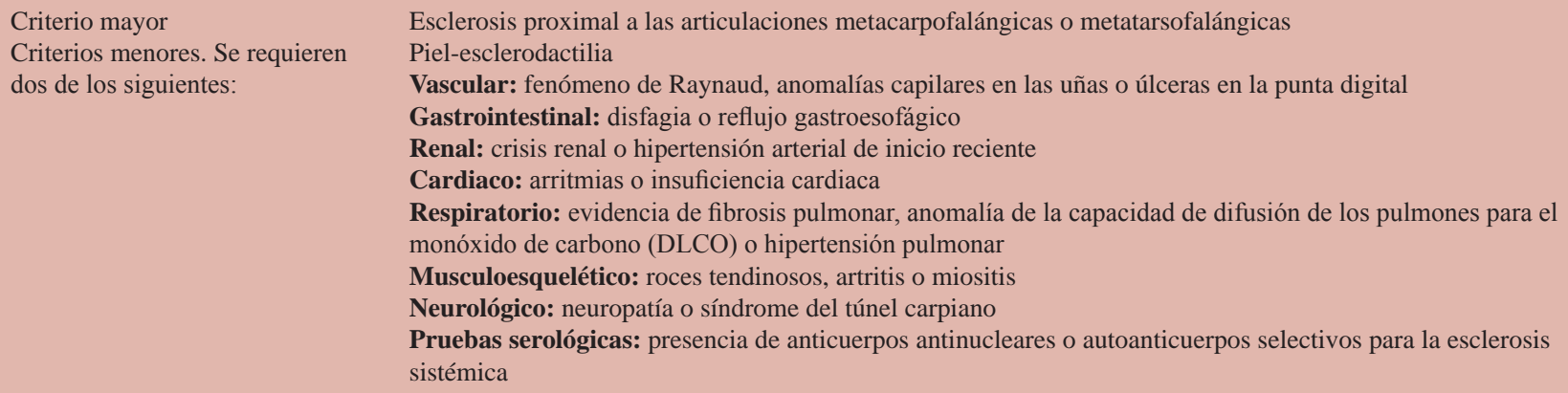

Esclerosis proximal a las articulaciones metacarpofalángicas o metatarsofalángicas Piel-esclerodactilia

Vascular: fenómeno de Raynaud, anomalías capilares en las uñas o úlceras en la punta digital Gastrointestinal: disfagia o reflujo gastroesofágico

Renal: crisis renal o hipertensión arterial de inicio reciente

Cardiaco: arritmias o insuficiencia cardiaca

Respiratorio: evidencia de fibrosis pulmonar, anomalía de la capacidad de difusión de los pulmones para el monóxido de carbono (DLCO) o hipertensión pulmonar

Musculoesquelético: roces tendinosos, artritis o miositis

Neurológico: neuropatía o síndrome del túnel carpiano

Pruebas serológicas: presencia de anticuerpos antinucleares o autoanticuerpos selectivos para la esclerosis sistémica

PRES = Consenso de Reumatología Pediátrica Sociedad Europea, ACR = Colegio Americano de Reumatología, EULAR = Liga Europea Contra el Reumatismo. 
Tabla 3: Esclerosis sistémica juvenil puntuación de gravedad

\begin{tabular}{|c|c|c|c|c|c|c|}
\hline & 0 (normal) & 1 (leve) & 2 (moderado) & 3 (severo) & 4 (etapa final) & $\begin{array}{c}\text { Puntaje máximo } \\
\text { posible }\end{array}$ \\
\hline General* & $\begin{array}{l}\text { IMC } \geq \text { línea de } \\
\text { base } \\
\mathrm{Hb}>11.5 \mathrm{~g} / \mathrm{dL}\end{array}$ & $\begin{array}{c}\mathrm{IMC}<1^{\mathrm{O}} \\
\text { percentil } \\
\mathrm{Hb} 10 \text { a } 11.4 \\
\mathrm{~g} / \mathrm{dL}\end{array}$ & $\begin{array}{c}\mathrm{IMC}<2^{\circ} \\
\text { percentil } \\
\mathrm{Hb} 9 \text { a } 9.9 \text { g/dL }\end{array}$ & $\begin{array}{c}\mathrm{IMC}<3^{\circ} \\
\text { percentil } \\
\mathrm{Hb} 7 \text { a } 8.9 \mathrm{~g} / \mathrm{dL}\end{array}$ & $\begin{array}{c}\mathrm{IMC}<4^{\circ} \\
\text { percentil } \\
\mathrm{Hb}<7 \mathrm{~g} / \mathrm{dL}\end{array}$ & 4 \\
\hline Vascular & No Raynaud & $\begin{array}{l}\text { Raynaud } \\
\text { requiere vasodi- } \\
\text { latadores }\end{array}$ & $\begin{array}{l}\text { Cicatrices de } \\
\text { punta digital }\end{array}$ & $\begin{array}{l}\text { Ulceraciones } \\
\text { digitales de la } \\
\text { punta }\end{array}$ & $\begin{array}{l}\text { Gangrena } \\
\text { digital }\end{array}$ & 4 \\
\hline Piel & MRSS 0 & MRSS 1 a 14 & MRSS 15 a 29 & MRSS 30 a 39 & MRSS $>40$ & 4 \\
\hline Osteoarticular & $\begin{array}{l}\text { No involucra- } \\
\text { miento articular }\end{array}$ & & $\begin{array}{l}\text { Presencia de } \\
\text { rango de movi- } \\
\text { miento limitado }\end{array}$ & & $\begin{array}{l}\text { Presencia de } \\
\text { artritis y/o fric- } \\
\text { ción tendinosa }\end{array}$ & 2 \\
\hline Músculo & $\begin{array}{l}\text { Fuerza mus- } \\
\text { cular proximal } \\
\text { normal }\end{array}$ & CMAS 39 a 51 & CMAS 26 a 38 & CMAS 13 a 25 & CMAS 0 a 12 & 2 \\
\hline GI* & $\begin{array}{c}\text { Resultados } \\
\text { normales de las } \\
\text { investigaciones } \\
\text { del tracto GI } \\
\text { proximal }\end{array}$ & $\begin{array}{l}\text { Síntomas GI } \\
\text { Hipomotilidad } \\
\text { esofágica distal } \\
\text { ERGE en } \\
\text { pHmetría o } \\
\text { cintiscan de } 24 \\
\text { horas }\end{array}$ & $\begin{array}{l}\text { Hipomotilidad } \\
\text { esofágica media } \\
\text { y/o alta }\end{array}$ & $\begin{array}{l}\text { Síndrome de } \\
\text { malabsorción }\end{array}$ & $\begin{array}{l}\text { Hiperalimen- } \\
\text { tación }\end{array}$ & 4 \\
\hline Respiratorio ${ }^{\S}$ & $\begin{array}{c}\text { DLco }>80 \% \\
\text { FVC }>80 \% \\
\text { Normal HRCT } \\
\text { sPAP }<30 \\
\text { mmHg }\end{array}$ & $\begin{array}{l}\text { DLco } 70 \text { a } 79 \% \\
\text { FVC } 70 \text { a } 79 \% \\
\text { Cambios de } \\
\text { vidrio molido } \\
\text { en HRCT } \\
\text { sPAP } 31 \text { a } 45 \\
\text { mmHg }\end{array}$ & $\begin{array}{c}\text { DLco } 50 \text { a } 69 \% \\
\text { FVC } 50 \text { a } 69 \% \\
\text { Cambios de } \\
\text { nido de abeja en } \\
\text { HRCT } \\
\text { sPAP } 46 \text { a } 75 \\
\text { mmHg }\end{array}$ & $\begin{array}{c}\text { DLco }<50 \% \\
\text { FVC }<50 \% \\
\text { Fibrosis en la } \\
\text { radiografía } \\
\text { sPAP }>75 \\
\text { mmHg }\end{array}$ & Dependencia $\mathrm{O}_{2}$ & 8 \\
\hline Cardiaco $^{\S}$ & $\begin{array}{l}\text { Normal ECG } \\
\text { FEVI }>50 \%\end{array}$ & $\begin{array}{c}\text { Defecto de } \\
\text { conducción de } \\
\text { ECG } \\
\text { LVEF } 45 \text { a } 49 \%\end{array}$ & $\begin{array}{c}\text { Arritmia } \\
\text { LVEF } 40 \text { a } 44 \%\end{array}$ & $\begin{array}{c}\text { Arritmia que } \\
\text { requiere trata- } \\
\text { miento } \\
\text { FEVI } 30 \text { a } 39 \%\end{array}$ & $\begin{array}{c}\text { Insuficiencia } \\
\text { cardiaca con- } \\
\text { gestiva } \\
\text { FEVI < 30\% }\end{array}$ & 8 \\
\hline Renal & $\begin{array}{c}\mathrm{GFR}>90 \mathrm{~mL} / \\
\text { minuto }\end{array}$ & $\begin{array}{l}\text { GFR } 75 \text { a } 89 \\
\mathrm{~mL} / \text { minuto }\end{array}$ & $\begin{array}{l}\text { GFR } 50 \text { a } 74 \\
\mathrm{~mL} / \text { minuto }\end{array}$ & $\begin{array}{l}\text { GFR } 10 \text { a } 49 \\
\text { mL/minuto }\end{array}$ & $\begin{array}{l}\text { Insuficiencia } \\
\text { renal terminal }\end{array}$ & 4 \\
\hline \multicolumn{7}{|c|}{ 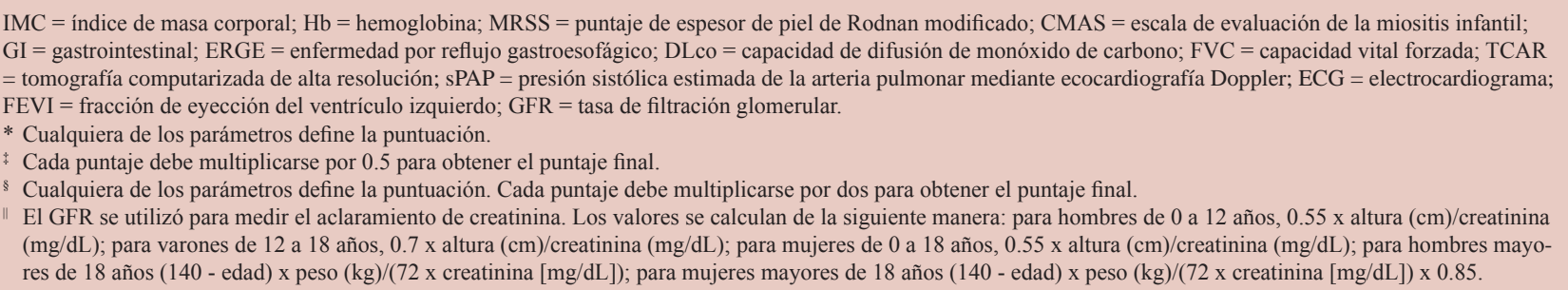 } \\
\hline
\end{tabular}

pueden desarrollar efectos adversos que requieren monitoreo adicional como hipomagnesemia y deficiencia de vitamina B12. La paciente se mantiene con las medidas no farmacológicas, ya que no presenta síntomas gastrointestinales al momento. ${ }^{6,8}$

En relación al pronóstico, el estudio de Martini y colaboradores, evaluó los factores que afectan la supervivencia en la ESJc en ciento treinta y cuatro pacientes en quienes se evidenció que se puede tener dos evoluciones posibles: la primera se presenta en pocos niños con un rápido desarrollo de insuficiencia orgánica interna que conduce a una discapacidad severa y la muerte, en la segunda los pacientes experimentan un curso lento e insidioso de la enfermedad con menor mortalidad, esta opción se relacionó con afectación cutánea limitada, pero también en una gran proporción de pacientes con el subtipo cutáneo 
difuso, sin inicio grave, en el que se evidenció un curso leve y buen pronóstico. ${ }^{9}$

\section{CONCLUSIONES}

La Esclerodermia Sistémica Juvenil es una patología rara que por lo general se presenta al final de la infancia, la afectación cutánea y sistémica llega a ser incapacitante e incluso mortal. La importancia de este caso es su presentación en una lactante de 13 meses de edad, con un inicio temprano y severo, por lo que su pronóstico es reservado; sin embargo, con el tratamiento multidisciplinario, al momento ha presentado remisión parcial de la sintomatología y el compromiso sistémico no ha involucrado otro órgano.

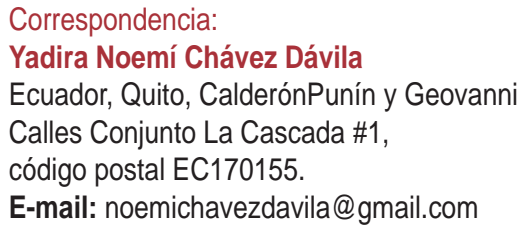

\section{BIBLIOGRAFÍA}

1. Pope JE, Johnson SR. New classification criteria for systemic sclerosis (scleroderma). Rheum Dis Clin North Am. 2015; 41 (3): 383-398.

2. HerrickAL, Ennis H, Bhushan M, Silman AJ, Baildam EM. Incidence of childhood linear scleroderma and systemic sclerosis in the UK and Ireland. Arthritis Care Res (Hoboken). 2010; 62 (2): 213-218.

3. Agarwal SK, Tan FK, Arnett FC. Genetics and genomic studies in scleroderma (systemic sclerosis). Rheum Dis Clin North Am. 2008; 34 (1): 17-40; v.

4. Salehzadeh F. A useful clinical sign in pediatric systemic sclerosis. J Clin Rheumatol. 2017; 23 (2): 113.

5. Li SC. Scleroderma in children and adolescents: localized scleroderma and systemic sclerosis. Pediatr Clin North Am. 2018; 65 (4): 757-781. doi: 10.1016/j.pcl.2018.04.002.
6. Zulian F, Balzarin M, Birolo C. Recent advances in the management of juvenile systemic sclerosis. Expert Rev Clin Immunol. 2017; 13 (4): 361-369. doi: 10.1080/1744666X.2017.1243467.

7. Adrovic A, Şahin S, Barut K, Kasapçopur Ö. Juvenile scleroderma: a referral center experience. Arch Rheumatol. 2018; 33 (3): 344-351.

8. Denton CP, Khanna D. Systemic sclerosis. Lancet. 2017; 390 (10103): 1685-1699. doi: 10.1016/S0140-6736(17)30933-9.

9. Martini G, Vittadello F, Kasapçopur O, Magni-Manzoni S, Corona $\mathrm{F}$, Duarte-Salazar $\mathrm{C}$ et al. Factors affecting survival in juvenile systemic sclerosis. Rheumatology (Oxford). 2009; 48 (2): 119122. 